\title{
Allogeneic Hematopoietic Cell Transplant for Patients with End Stage Renal Disease Requiring Dialysis - a Single Institution Experience
}

\author{
Mazyar Shadman, MD, MPH ${ }^{1,2}$, Sangeeta Hingorani, MD, MPH ${ }^{1,3}$, Scott A. Lanum, Pharm \\ $\mathrm{D}^{4}$, John M. Pagel, MD, PhD ${ }^{5}$, Rainer Storb, $\mathbf{M D}^{1,2}$, David G. Maloney, MD, $\mathbf{P h D}^{1,2}$, and \\ Brenda M. Sandmaier, MD ${ }^{1,2}$ \\ ${ }^{1}$ Clinical Research Division, Fred Hutchinson Cancer Research Center, Seattle, WA \\ ${ }^{2}$ Department of Medicine, University of Washington, Seattle, WA \\ ${ }^{3}$ Department of Pediatrics, University of Washington, Seattle, WA \\ ${ }^{4}$ Department of Pharmacy, University of Washington, Seattle, WA \\ ${ }^{5}$ Swedish Cancer Institute, Seattle, WA
}

\section{Keywords}

Allogeneic hematopoietic stem cell transplant; End Stage Renal Disease; Dialysis

\begin{abstract}
Allogeneic hematopoietic cell transplantation (allo-HCT) is a potentially curative treatment for a number of benign and malignant hematologic disorders; however, it is also associated with treatment-related complications. ${ }^{1}$ The advent of reduced-intensity (RIC) and nonmyeloablative (NMA) conditioning regimens has made allogeneic HCT a viable option for patients who might otherwise have been ineligible because of medical comorbidities. ${ }^{2}$ Patients with hematologic malignancies are at increased risk of developing renal insufficiency either because of the disease pathophysiology (e.g. myeloma) or as a result of adverse effects from treatment with multiple lines of cytotoxic chemotherapy agents. ${ }^{3}$ Unlike autologous HSCT, the published data regarding the feasibility and outcomes of alloHCT for patients with chronic kidney disease (CKD) and especially for those with end-stage renal disease (ESRD) requiring renal replacement therapy is limited. This paucity of data may lead to the unnecessary exclusion of patients on hemodialysis or peritoneal dialysis from allogeneic-HCT as a treatment option. Herein, we report the outcomes of patients with hematologic malignancies who were transplanted while on renal replacement therapy (RRT) at our institution. In this retrospective study, we used our institutional database at Fred Hutchinson Cancer Research Center (FHCRC) and reviewed all patients who received a RIC or NMA allo-HSCT from 1997 to 2014. For this report we included the patients who were on either hemodialysis or peritoneal dialysis at the time of transplant at the FHCRC. Clinical data were extracted from electronic medical records directly. The study is approved by the FHCRC IRB. We identified 6 patients who were transplanted at the FHCRC between 1997
\end{abstract}


and 2014 while on dialysis. Their median age was 43.5 (range $13-52$ years) and $50 \%$ of patients were female $(n=3)$. (Table-1) Multiple myeloma was the most common etiology for ESRD in 3 patients followed by polycystic kidney disease (PCKD) in 2 and hemolyticuremic syndrome (HUS) secondary to E.coli infection in 1. The median duration of receiving RRT before HSCT was 14 months (range: $6-48$ months). Five patients were on hemodialysis (HD) and 1 was on peritoneal dialysis (PD). Primary hematologic disorders included multiple myeloma $(n=3)$, follicular lymphoma $(n=1)$, myelodysplastic syndrome (MDS) $(n=1)$ and Wiskott-Aldrich syndrome $(n=1)$. Four of the 6 patients had previously undergone an autologous HCT. Three patients received their peripheral stem cell grafts from HLA-matched-related donors. For these patients, conditioning included $2 \mathrm{~Gy}$ total-body irradiation (TBI) at a rate of $0.07-0.10 \mathrm{~Gy} /$ minute from a linear accelerator on the day of HCT (day 0). Two patients had peripheral stem cell grafts from HLA-matched-unrelated donors after conditioning with fludarabine ( $30 \mathrm{mg} / \mathrm{m}^{2} /$ day days -4 to -2$)$ in addition to 2 Gy TBI (day 0). Fludarabine was given at full dose in one patient who was on HD at the time of transplant consult but not at the time of conditioning. In the other patient, the dose was reduced by $25 \%$ and the drug was given after $\mathrm{HD}$ in each day of administration. One patient received an HLA-haploidentical bone marrow transplant. The conditioning protocol included full-dose cyclophosphamide $(14.5 \mathrm{mg} / \mathrm{kg} / \mathrm{day}$ on days -6 and -5$)$, fludarabine dose reduced by $50 \%$ (to $15 \mathrm{mg} / \mathrm{m}^{2} /$ day on days -6 to -2 ) and 2-Gy TBI (day -1). Both cyclophosphamide and fludarabine were given after dialysis on each treatment day. Patients with HLA-matched related or unrelated grafts $(\mathrm{n}=5 ; 83 \%)$ received cyclosporine and mycophenolate mofetil (MMF) for GVHD prophylaxis. Cyclosporine dose was adjusted to achieve a therapeutic level between $120-360 \mathrm{ng} / \mathrm{mL}$ as tolerated by toxicity. MMF was given at the standard dose. The GVHD prophylaxis regimen for the patient who received an HLAhaploidentical transplant included a single dose of cyclophosphamide $50 \mathrm{mg} / \mathrm{kg}$ on day 3 after HCT, which was given with $25 \%$ dose reduction $(37.5 \mathrm{mg} / \mathrm{kg}$ ) after dialysis. In addition, the patient received tacrolimus (starting day +4 ) and MMF. Tacrolimus dosing was adjusted to achieve blood level between 5-15 ng/ml. After median follow-up of 15.5 months (range: 9.6-84.6), one patient was alive with no evidence of recurrence of multiple myeloma 24 months after HLA haploidentical transplant. Five patients (83\%) died between 9.6 to 84.5 (median 12.2 months) months after transplant. One patient died at 9.6 months from tumor lysis syndrome after disease relapse. The remaining patients died from etiologies other than relapse: one died at 10.7 months from CMV pneumonia, one at 12.3 months from cerebral hemorrhage secondary to fungal infection, one from uncontrolled bleeding during cardiac surgery for aortic aneurism. Bleeding in this patient was not related to renal failure. One patient died at 84.7 months from sepsis. We did not observe any of the known serious complications of calcineurin inhibitors like posterior reversible encephalopathy syndrome (PRES) or thrombotic microangiopathy (TMA) in our patients. Six patients who were on either hemodialysis or peritoneal dialysis while undergoing allogeneic HCT for advanced blood disorders did not experience adverse short-term or long-term events related to their end-stage renal disease or renal replacement therapy. While meticulous clinical monitoring and dosing adjustment for both conditioning regimen and post-transplant GVHD medications is necessary in these patients, they should not be routinely excluded from allogeneic HCT solely because they are on renal replacement therapy. In a recent comprehensive review, Bodge and colleagues ${ }^{4}$ discussed the recommendations for optimal 
adjustments of preparative regimens in patients with different stages of chronic kidney disease. Experience is limited in regards to allogeneic-HCT for end-stage renal disease patients requiring dialysis and clinical practices have been quite variable. ${ }^{5-7}$ Fludarabinebased regimens in combination with low dose TBI are commonly used for NMA allogeneic HCTs. It has been shown that approximately $60 \%$ of the active metabolite of fludarabine is eliminated by the kidneys and therefore appropriate dose adjustment needs to be implemented in patients with renal failure. ${ }^{8}$ In addition, data on dialysate concentration and pharmacokinetics of the principal metabolite of fludarabine, 2-fluoro-ara-A (2F-Ara-A) suggests that fludarabine is adequately removed by hemodialysis and can be used in dialysis patients with dose adjustment. ${ }^{9}$ We administered dose-adjusted fludarabine after dialysis and consistent with our approach, other groups have reported use of fludarabine with $20 \%$ dose reduction in dialysis patients. ${ }^{6-7}$ In one report, 4 of 5 patients with different degrees of renal failure developed severe neurologic toxicities including 2 deaths when transplanted after conditioning with fludarabine-based regimens. ${ }^{7}$ Of note, in their report, the only patient without neurotoxicity was one of the 2 dialysis dependent patients who in fact received full dose fludarabine ( $30 \mathrm{mg} / \mathrm{m} 2$ for 5 days). Calcineurin inhibitors and/or late effect of fludarabine were reported as possible etiologies for neurotoxicity and significant dose reduction or even omission of fludarabine from conditioning regimens for patient with renal failure was suggested by the authors. As reported above, we did not observe any neurologic adverse events in our patients and are reluctant to agree with their recommendations in the absence of stronger evidence for neurotoxicity by fludarabine. Cyclophosphamide and its metabolites are also cleared by the kidneys and only partially removed by dialysis. There are no universally recognized guidelines for dosing of cyclophosphamide in patients with renal impairment and in those on dialysis. Optimal dose adjustment remains controversial for cyclophosphamide. It is in general recommended that cyclophosphamide doses be reduced by $25-50 \%$ in patients with severe renal impairment (GFR $<10 \mathrm{~mL} / \mathrm{min}$ ) and some authors suggest a supplemental dose after dialysis. ${ }^{10-12}$ As a result, in published case reports of dialysis patients undergoing allogeneic-HCT with cyclophosphamide containing regimens the approach is variable and inconsistent. In addition, timing of the first dialysis after cyclophosphamide has been variable ranging from 6 to 14 hours. ${ }^{13-14}$ In our patients, the lower doses $(14.5 \mathrm{mg} / \mathrm{kg} / \mathrm{day}$ on each of 2 successive days) of cyclophosphamide in the conditioning regimen were not changed; however, the higher dose $(50 \mathrm{mg} / \mathrm{kg})$ cyclophosphamide scheduled for day 3 after transplant used as GVHD prophylaxis was reduced by $25 \%$. In both instances, the drug was given after dialysis. Dialysis does not appreciably alter the pharmacokinetics of cyclosporine, tacrolimus, or MMF. ${ }^{10}$ For calcineurin inhibitors dosing was done according to trough levels which resulted in less frequent administration of drugs. We have demonstrated the ability to successfully transplant patients on dialysis and suggest that dialysis should not be an exclusion criterion. The current report only includes patients with history of end-stage kidney disease who were established dialysis patients at the time of referral to our transplant center. Systematic review of national registries is needed in order to address the feasibility and safety of allogeneicHCT in ESRD patients requiring dialysis. 


\section{Supplementary Material}

Refer to Web version on PubMed Central for supplementary material.

\section{Acknowledgments}

Funding: CA078902, CA18029, CA15704, HL122173

\section{References}

1. Majhail NS, Farnia SH, Carpenter PA. Indications for Autologous and Allogeneic Hematopoietic Cell Transplantation: Guidelines from the American Society for Blood and Marrow Transplantation. Biol Blood Marrow Transplant. 2015; 21:1863-9. [PubMed: 26256941]

2. Baron F, Storb R. Allogeneic hematopoietic cell transplantation following nonmyeloablative conditioning as treatment for hematologic malignancies and inherited blood disorders. Mol Ther. 2006; 13:26-41. [PubMed: 16280257]

3. Niscola P, Vischini G, Tendas A, et al. Management of hematological malignancies in patients affected by renal failure. Expert Rev Anticancer Ther. 2011; 11:415-32. [PubMed: 21417855] Bodge MN, Reddy S, Thompson MS, et al. Preparative Regimen Dosing for Hematopoietic Stem Cell Transplantation in Patients with Chronic Kidney Disease: Analysis of the Literature and Recommendations. Biol Blood Marrow Transplant. 2014; 20:908-919. [PubMed: 24565993]

4. Hamaki T, Katori H, Kami M, et al. Successful allogeneic blood stem cell transplantation for aplastic anemia in a patient with renal insufficiency requiring dialysis. Bone Marrow Transplant. 2002; 30:195-198. [PubMed: 12189539]

5. Horwitz ME, Spasojevic I, Morris A, et al. Fludarabine-based nonmyeloablative stem cell transplantation for sickle cell disease with and without renal failure: clinical outcome and pharmacokinetics. Biol Blood Marrow Transplant. 2007; 13:1422-1426. [PubMed: 18022571]

6. van Besien K, Schouten V, Parsad S, et al. Allogeneic stem cell transplant in renal failure: engraftment and prolonged survival, but high incidence of neurologic toxicity. Leuk Lymphoma. 2012; 53:158-159. [PubMed: 21749304]

7. Lichtman SM, Etcubanas E, Budman DR, et al. The pharmacokinetics and pharmacodynamics of fludarabine phosphate in patients with renal impairment: a prospective dose adjustment study. Cancer Invest. 2002; 20:904-913. [PubMed: 12449721]

8. Kielstein JT, Stadler M, Czock D, et al. Dialysate concentration and pharmacokinetics of 2F-Ara-A in a patient with acute renal failure. Eur J Haematol. 2005; 74:533-534. [PubMed: 15876260]

9. Haubitz M, Bohnenstengel F, Brunkhorst R, et al. Cyclophosphamide pharmacokinetics and dose requirements in patients with renal insufficiency. Kidney Int. 2002; 61:1495-1501. [PubMed: 11918757]

10. Aronoff, GR., Bennett, WM., Berns, JS., et al. Drug Prescribing in Renal Failure: Dosing Guidelines for Adults and Children. 5. Philadelphia, PA: American College of Physicians; 2007. p. 97-170.

11. Janus N, Thariat J, Boulanger H, et al. Proposal for Dosage Adjustment and Timing of Chemotherapy in Hemodialyzed Patients. Ann Oncol. 2010; 21:1395-403. [PubMed: 20118214]

12. Bischoff ME, Blau W, Wagner T, et al. Total body irradiation and cyclophosphamide is a conditioning regimen for unrelated bone marrow transplantation in a patient with chronic myelogenous leukemia and renal failure on hemodialysis. Bone Marrow Transplant. 1998; 22:591593. [PubMed: 9758350]

13. Perry JJ, Fleming RA, Rocco MV, et al. Administration and pharmacokinetics of high-dose cyclophosphamide with hemodialysis support for allogeneic bone marrow transplantation in acute leukemia and end stage renal disease. Bone Marrow Transplant. 1999; 23:839-842. [PubMed: 10231150] 


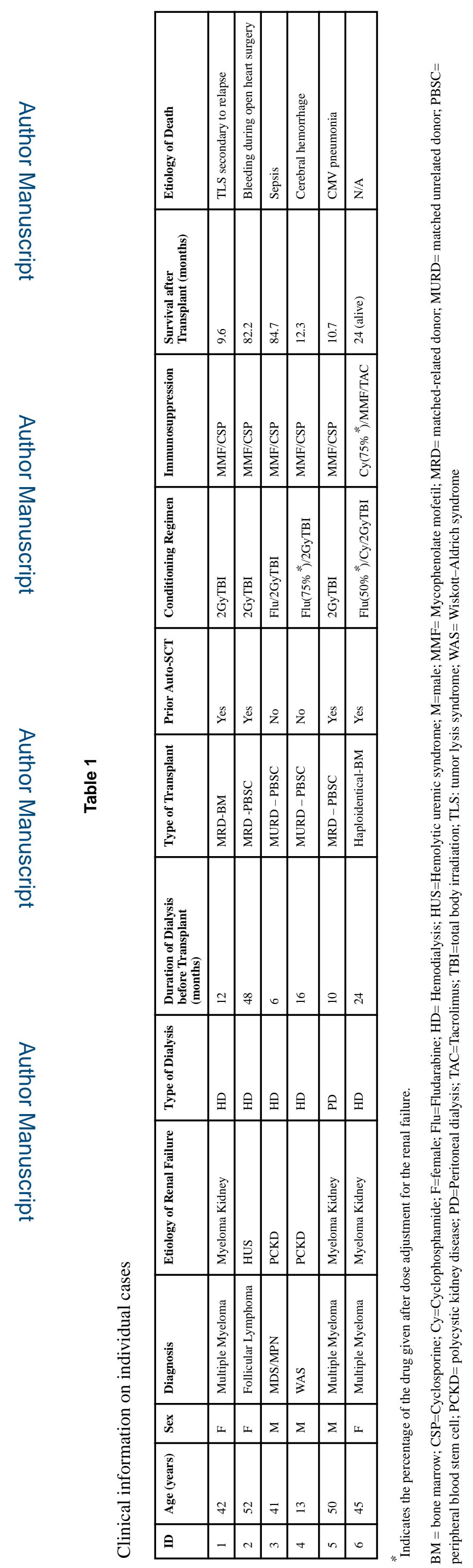

